

\title{
1767: POR QUÉ LOS JESUITAS
}

\author{
POR \\ JOSÉ ANDRÉS-GALLEGO \\ Hispania Sacra
}

\section{RESUMEN}

Exposición ordenada de los argumentos antijesuíticos y antipapales de Bernardo Tanucci, ministro de Carlos III en Nápoles y regente de este reino.El autor cree que la expulsión de los jesuitas de la monarquía española se comprende al conocer la idea que se tenía de ellos.

\section{ABstract}

\section{7: Why the Jesuits}

Study about Bernardo Tanucci's arguments in relation to the Jesuits and the Pope, when Tanucci was regent of Napoles. According the author, Jesuits expulsion from the Spanish Monarchy can be understood knowing the idea that Carlos III and Tanucci had about them.

En el estudio que nos ocupa desde hace años sobre la mentalidad política hispana, centrado en los años sesenta del siglo XVIII, la expulsión de los jesuitas aparece una y otra vez como un acontecimiento fundamental y, al tiempo, como una gran incógnita. ¿Por qué los jesuitas?

Se han aducido muchas causas, y la sistematización de las mismas nos ocupará en las primeras páginas. Pero - todas juntas - no bastan para explicar la magnitud de la medida adoptada en 1767 . Confieso que no he comprendido las razones de lo que hizo Carlos III hasta leer la correspondencia de Tanucci, que viene siendo publicada en una muy notable edición crítica. La 
presentación y ordenación de sus argumentos, de modo que se advierta su enorme envergadura, en el propósito de estas páginas.

Leyendo al toscano, sí se entiende la expulsión. Sencillamente, si Carlos III y sus consejeros pensaban lo que pensaba el miembro de la regencia napolitana acerca de la Compañía de Jesús y de la propia Iglesia, el asunto es más que inteligible: es lógico. La Compañía se percibía como una realidad maligna hasta un extremo que sólo la lectura de esos textos permite comprender. Esa será la parte gruesa de estas páginas.

Pero no querría dejar de lado que, a su vez, la concepción de la Compañía de Jesús, en Tanucci, estaba íntimamente ligada a la concepción de la Iglesia. Y ésta nada tenía que ver con la eclesiología que habían definido los teólogos escolásticos. Era una eclesiología marcadamente impregnada del espiritualismo protestante, en aquellas versiones que desechaban la existencia de la Iglesia fuera del ámbito de la conciencia.

La Iglesia está en el Estado,'no sobre el Estado. La afirmación -..en un católico- es importante porque ayuda a entender lo que se diría después: en el siglo XIX y en el XX, hasta hoy mismo.

En otras palabras: la negación —que hoy aún se escucha - de la personalidad internacional de la Sede Apostólica está ahí en germen: en una concepción eclesiológica protestante que se había filtrado en el catolicismo sobre todo en el siglo XVIII, hasta hoy.

\section{CAUSAS DE LA EXPULSIÓN: LA ENEMIGA A LOS JESUITAS ${ }^{\prime}$}

¿Por qué los jesuitas? Ya se ha apuntado la enemiga a los religiosos que había en los más de los regalistas del XVIII, que los consideraban como meros haraganes inútiles. Pues bien, entre ellos, ningunos tan nocivos como los de la Compañía de Jesús. Cuya silueta tenía ciertamente la extraña virtud de producir los más ardorosos afectos y los más fuertes odios. "Los superiores de Méjico -escribía, entre tantos, el jesuita autor de las litterae annuae de 1757 sobre las misiones septentrionales de Nueva España - [...] ya han experimentado a menudo la facilidad con que se levantan contra los nuestros las más enormes imposturas"2 . Al acordar el extrañamiento —aduciría por el contrario Aranda en 1767, refiriéndose a las reducciones jesuíticas-, "se tuvieron

I Salvo que se indique algo en contra, para todos los aspectos relacionados con la bibliograffa sobre el tema remitimos a POLGAR (1985 y 1986); en lo que concierne concretamente a las misiones, ved además Bibliographia missionaria (1937-), anuario publicado en la Biblioteca Misional Pontificia de la Congregación para la Evangelización de los Pueblos, Ciudad del Vaticano.

2 Apud BURRus (1963) 23.

La Personalidad Internacional de los Papas

Hispania Sacra 48 (1996) 
presentes los vicios espirituales como los temporales de que adolecen y las maniobras con que dichos Padres, afectando en lo exterior dimitirlas, han ganado varios obispos y aun tribunales para acreditarse de necesarios y parecer tales" $"$.

Meses después de la expulsión, cuando el secretario de Gracia y Justicia, don Manuel de Roda, informara acerca de la ejecución del extrañamiento de los jesuitas del Paraguay por parte del gobernador de Buenos Aires, don Francisco de Bucareli, en 1768, daría buena muestra de este tipo de atribuciones. Cuya reiteración, sin embargo, fuerza a pensar que se creía verosímil y que, por tanto, aunque no respondiera a la verdad, podía servir para justificar la decisión de acabar con ellos: aquéllos indios, dice Roda, "eran esclavos que obedecían ciegamente lo que los padres jesuitas les mandaban." Y eso por más que, según él, los trataban de la peor manera que se pudiera imaginar: no los confesaban - aseguraba - ni les daban el viático en sus casas cuando estaban enfermos, sino que - "por no incomodarse" - los obligaban a ir a recibirlo a un lugar cercano a la iglesia y los dejaban morir sin más auxilio ni asistencia; "incorregigles en sus excesos", administraban los sacramentos de un modo contrario a la práctica de la Iglesia católica y, en concreto, a las indicaciones que les había hecho el obispo de Buenos Aires; no se preocupaban de evangelizar a los indígenas infieles más próximos a las reducciones, no pocos de los cuales - aseguraba Roda - se habían presentado ante los nuevos administradores eclesiásticos para bautizarse y reducirse en cuanto tuvieron noticia de que se habían ido los padres. "No han sido ocultos los objetos de aquella depravada norma", añadía, con una afirmación que es una incógnita.

Pese a la fertilidad del país, mantenían a los guaraníes en la mayor miseria; sólo les daban una escasa ración de carne y un poco de maíz como toda comida y, sólo al padre de familia, una onza de yerba. Y lo mismo con el vestido, en las mujeres "con un extremo que no puede declararse sin faltar a la modestia". "Estas tiranías y la estrechez de las habitaciones ocasionaban muchas ofensas a Dios, y frecuentes enfermedades y epidemias, en que perecía crecido número de aquellos pobres a cuyas expensas engrandecían los jesuitas su poder, disfrutando toda la substancia de este imperio; que así lo titulaban comúnmente."

No les habían dejado aprender castellano ni rozarse con español alguno, y eso a pesar de las varias órdenes emanadas del rey para que se pusieran escuelas donde pudieran aprenderlo. "Constante ha sido el despotismo con que los han dominado, sin permitierles conocer más Dios, Rey, ni santos, que a los de

3 Al marqués de San Juan, 13 de abril de 1767, AGl, tndiferente general, 3.085-A.

La Personalidad Internacional de los Papas Hispania Sacra 48 (1996) 
la Compañía, teníendoles infundido en el corazón un odio implacable contra los españoles"4.

Todo ello no era en realidad sino un aspecto de la sed de poder que se les achacaba. Un instrumento principal habían sido los confesores regios: Daubenton y Rávago en la época de Felipe V y Fernando VI respectivamente; antes, el padre Nithard y los de los validos de los Austrias; incluso los de los hijos de Carlos III, que desde 1748 había confiado a jesuitas su educación y orientación moral, y los de su madre y su esposa ${ }^{5}$.

Il padre Ravago - escribía Tanucci a Losada al comenzar 1764-avrá dovuto render conto a Dio di gran cose, e di molti mali, che alla monarchia, e al suo padrone ha fatti per la sua Compagnia. Tutto gesuita ovunque si ponga fará lo stessot.

4 En su derrota, cuenta, el gobernador encontró al "famoso Nicolás [Nengurin] de quien tanto han hablado las gacetas", y Nengurin le explico la desgracia en que se encontraba desde hacía atoos por no haber ejecutado las ordenes - "por haberle parecido tiranía"- que le habla dado en su momento el padre José Carojiel y que consistieron en que pasara a cuchillo el destacamento de milicias del Paraguay; "que vino al monte grande, después del sacrificio tan ínhumano que allí mismo ejecutaron con el de los Correntinos, pareciéndole que cumplía con quitarles las armas y caballos, como lo hizo; y que inmediatamente le desposeyeron de cuanto tenía y lo desterraron desde el pueblo de la Concepción, de donde era natural, al de la Trinidad, en que había permanecido hasta ahora, que informado de que el gobernador estaba cerca pudo huirse y venir a encontrarte." Aquí, lo de que no créá tener responsabilidad -sigue Roda- "en atención a que eran esclavos que obedecían ciegamente lo que los padres jesuitas les mandaban."

En el Yapeyú, Bucareli se mantuvo diez días "para disipar las especies con que los de la Companía tenían engañados a los pobres indios"; consiguió que regresaran los que estaban huidos en chácaras y montes "a influjo de los jesuitas". En uno de los pueblos mús distantes, el cura de! mismo, Tadeo Enis, indujo a los indígenas a que exigieran al comisionado - en ese caso Riva Hertern, enviado por Bucareli- que mostrara la bula pontificia en virtud de la cual efectuabas el extrañamiento.

En Santo Tomé, el pueblo manifestó el sentimiento de que sus curas hubieran quemado "hasta las ráices de los árboles de la huerta y hecho otras acciones poco cristianas, sirviéndoles de consuclo su expulsión". Esto y lo citado arriba, apud FERRER (1990), 49-54.

Hay que decir que la influencia de los jesuitas de las reducciones sobre los indios para que no aceptaran el tratado de límites, la atestiguó el también jesuitz Lope Luis Altamirano, enviado para obligarles a cumplirlo: "Los indios —escribia a Rávago el 22 de julio de 1753- ya estarían mudados a haber querido seriamente los Padres, que en mi dictamen, según lo que he observado y experimentado, son la verdadera causa de su levantamiento y perdición, y del descrédito de nuestra buena madre la Compañia,...: apud ALCARAZ (1995), 689.

$s$ Sobre esto, MEDINA (1991), 16-7: El jesuita que se encargó de preceptuar al príncipe Carlos y a los infantes en 1748 fue el andaluz José de Barba y Guzmán; paso con ellos a España en 1759 y, debilitado por la edad, to sustituyó en 1762-1763 el gaditano y físico, jesuita también, Antonio Zacagnini.

6 Carta de 17 de enero de 1764, apud TANucCl (1994), 10

La Personalidad Internacional de los Papas

Hispania Sacra 48 (1996) 
Y de la regia cámara a todas las cámaras de la Monarquía Católica. En todos los niveles de gobierno, desde el general hasta el local, se les acusaba de intervenir y maniobrar para colocar sus peones. "Para llevar adelante sus ideas -escribirá, pongo por caso, el gobernador de Buenos Aires al conde de Aranda en 1768-, los de la Compañía solicitaron colocar en todos los empleos de estos reinos y provincias a los que ciegamente se entregaban a su disposición o que habían criado dependientes de su arbitrio, practicando los medios más reprobados a fin de mudar y destruir [a] los que juzgaban embarazo a sus máximas". Entre otras cosas, habían pensado en don Pedro Cevallos como virrey del Perú y luego como secretario de Indias ${ }^{7}$.

La propia caída de Rávago en 1755, vinculada a la del marqués de la Ensenada, de voluntad filojesuítica, había estado unida de hecho a la leyenda de las ambiciones de constituir un reino jesuítico en América $^{8}$. No otra había sido la razón - se argüía - del empeño puesto por los religiosos encargados de las reducciones guaraníes para frustrar el tratado de límites hispanoportugués de 1750. Porque ellos habían sido quienes azuzaran a los indígenas contra los nuevos ocupantes portugueses. Habían pretendido formar un gran ejército indígena para acabar también con la soberanía española y formar un imperio jesuítico -afirmaba el obispo de Tucumán, Abad e Illana-.. En 1755, se corría de hecho por Madrid que habían nombrado rey a un Nicolás I, que había labrado moneda con su efigie y algunas habían llegado a la corte; incluso el rey tenía tres...?.

Prácticas que, de hecho, se habrían concretado en la acumulación de enormes riquezas ${ }^{10}$. Que se buscarían con denuedo en 1767, al ocupar militarmente todas sus pertenencias, y aun mucho después (hasta hoy mismo).

Los privilegios que habían recibido tanto de los pontífices como de los monarcas, además, los procuraban defender y ampliar contra viento y marea, frente al criterio de obispos y superiores de otras órdenes y también de gobernantes civiles que veían mermada así su jurisdicción. Y ahí se inscribía el ejemplo reciente de la exención de diezmar en América ${ }^{11}$.

7 Cir. ACEVEDO (1969), 38.

8 Esta del tratado de límites de 1750 sólo fue una, en realidad, de jas posibles causas de la caída de Ensenada. En cuanto a Rávago, también pudo suceder así, pese a que había apoyado el trałado en cuestión: yid. ALCARAZ (1995), 679-84.

9 Vid. Alcaraz (1995), 705.

10 Sobre la presencia del mito de las riquezas jesuíticas inmediatamente antes de la expulsión, concretamente en tierras peruanas y en relacion con los misiones de mojos, ARSI, Peru., 18: Littere Annue... ab anno /761 in annum /765 [1766], f. 279. Ved la vision de conjunto de ARMANI (1982). Sobre la acusacion de haber formado un reino independiente, KRATZ (1954), 195-6.

11 Documentaci6n sobre el asunto del diezmo, entre otros lugares, en AGI, Indiferente general, 3.085-A y B; ARSI, Hist. Soc., 201-11, f, 127-136 (memorial del procurador general de los jesuitas a 
Al cabo ni más ni menos que todo eso era lo que los había enfrentado en el siglo XVII con el obispo Palafox, de La Puebla de los Angeles, quien se había atrevido a reconvenirlos y había sufrido como contrapartida la más feroz persecución. En vida y post mortem. Porque los jesuitas eran quienes, con su influencia en Roma, habían impedido e impedían que fuera beatificado.

Eran abanderados del laxismo teológico y, en relación con ello, defendían una moral de justificación de la mentira en determinadas situaciones. "El imperio de las conciencias con su laxa moral -certificaba Campomanes-, desconocida de los Santos Padres, y que Gravina creyó tan dañosa como herejía, fue inundando no sólo a España sino a todo el orbe católico, y a no mediar aquella alta providencia del que gobierna el universo, hubieran perpetuado estos hombres el dominio universal, a que estaban tan cercanos"'12.

Se les atribuía por lo tanto doblez, y eso no sólo en general sino aplicado a cualesquiera circunstancias. Los ingleses que ocuparon Manila en 1762 -relataba un fraile agustino en $1770^{13}$ - mataron a otros padres de otras religiones, a excepcion de la Compañía, porque "ésta hacía a dos caras, pues por un lado era del inglés y no se metió con ellos, y por otro del Sr. Anda, aunque éste se recelaba mucho de ellos." El oidor don Simón de Anda había, como sabemos, organizado la guerra contra los invasores británicos en el archipiélago.

Por lo demás, la desconfianza en su lealtad -consecuencia de la doblez que se les achacaba-- se había vuelto a agravar en 1766, a raíz del motín de Squillace, y así lo había puesto de relieve el fiscal Campomanes en la consulta que sirvió de argumento a la decisión de extrañarlos. Sólo que la ocasión la pintaron calva y sirvió para recordar las más antiguas quejas.

\footnotetext{
Carlos III sobre diezmos en Indias), y sobre todo ASV, passim. En el primero de los lugares mencionados, 3.085-A, vid. además la Copia de lo mós substancial del Testimonio dado por el Es [criba/no Joseph Pazniño, comprovado de otros tres, suflec]ha en la Cindad de Sloln Franlcis/co de Quito a 5 días de Abril de $1763 . .$. , acerca de las haciendas jesuitas. Sobre lo reducido del crecimiento en el arzobispado de Méjico, certificacion del bachiller don Juan Josef de Mier, 18 de abril de 1761, ibidem. Vid. también ARSI, Hist. Soc., 207, f. 142-169v, sobre ln oposici6n al tratado de línites, especialmente en 1752-1753; aunque ya se sabe que este asunto ha sido objeto de un muy amplio tratamiento, sobre todo de KRATZ (1954), también de MATEOS (1951-2) y desde el punto de vișta brasileño ESCANDON (1983). Una breve síntesis del asunto, en SANTOS (1992: 284-8).

12 Apud Barajas (1994), 523.

13. Relación sucinta, clara y veridica de la toma de Manila por la escuadra inglesa, escrita por el P. Fr. Agustín María de Castro y Amuedo..., apud Navarro (1908), 1, 87. En el mismo sentido, el fiscal Francisco Leandro Viana en su Diario del Siho..., ibidem, I, 421 y 487 . Otros detalles en diversos documentos, ibidem, II, 194 y 409-12 (dictamen del viceprovincial, Bernardo Pazuengos, sobre lo hecho por los jesuitas durante la guerra, 2 de septiembre de 1763).
}

La Personalidad Internacional de los Papas

Hispania Sacra 48 (1996) 
Y aparte estaba su fidelidad al papado frente a las regalías del monarca, allá donde estuvieran, fuera España u otra nación.

\section{LA VISIÓN DE TANUCCI: LOS DEFECTOS DE LOS JESUITAS Y LA CURIA DE ROMA}

Antes de preguntarnos si todo esto era verdad se ha de añadir otra cosa: en qué medida lo creía Carlos III. Porque eso fue lo decisivo. Y ante ello hay que decir que al rey se los habían pintado como verdaderos monstruos odiosos, que valía la pena extirpar, "ai quali non importa che tutto il mondo cattolico s'infiammi, e vada in rovina, purché la Compagnia ingrassi, e vinca tutti $i$ suoi punti, anche quelli di scuola per li quali non hanno ribrezzo di suscitar incendi fatali alle nazioni cattoliche, e alle vite delli stessi sovrani"]4,

A ellos se debía la tendencia de todo súbdito a robar a su príncipe.

Li teologi gesuiti hanno prodotto questo male con dottrine diaboliche, e scelerate. Ah li sovrani non hanno tempo di studiare, se lo avessero non sarebbono $[. .$.$] sovrami$ che li volessero ne loro statils.

Un corpo di gente sparsa tra le nazioni coll'obbligo d'una cieca obbedienza in tutte le loro azioni al Generale e sciolta da tutte le leggi é tun corpo d'atei della sovranitá, un esercito di banditi o di corsari che investe ognuno, e insoffribile nella societá unana colla quale professa di non aver alcun vincolo. I Liberi Muratori son piu soffiribili perché non possiedono né usurpano li beni dei cittadini di quelle nazioni ove stanno. Si vede una gran relazione tra le costituzione, e la morale ${ }^{16}$.

Ellos eran también quienes habían usurpado las funciones de los clérigos seculares: "Le scuole, le confraternite, e le missione dovrebbono esser tolte ai Geuisti nell'universo mondo, e la confessione ancora, e gli esercizi spirituali. Tutte queste incombenze son de' preti secolare. Se $i$ Gesuiti vogliono stare

14 A Wall, 15 de septiembre de 1761, apud TanuCCl (1988), X, I 18 . En los párafos que siguen hacemos un estudio de la correspondencia de Tanucci, que casi nunca dice estas cosas directamente al rey Carlos III, sino a los demás. Pero llama la atención el hecho de que los textos más explícitos los dirija al duque de Losada y a Wall, los hombres más cercanos al monarca en aquellos momentos. Algunos giros inducen a pensar que les hablabn asi para que se lo trasmitiesen al rey como opinión propia o ajena. En una de las cartas más explícita y prolijamente antijesútica, la del 3 de noviembre de 1761 que dirige al duque de Losada, Tanucci acaba pidiéndole perdón por la largura del discurso, "che non si può né si deve fare se non ad un soggetto quale e Vostra Eccellenza prossimo ad un sovrano, $e$ confidente d'un sovrano": ibidem (1988), X, 260.

15 A Squillace, 22 de septiembre de 1761, ibidem (1988), X, I5I.

16 A Pignatelli, 29 de septiembre de 1761, ibidem (1988), X, I6I. 
uniti in conventi, cantino mattutini, vespri, e messe, si dieno la disciplina $e$ nulla facciano di secolaresco e mondano" ${ }^{\prime 17}$.

No faltaba el delito del laxismo moral:

Uomini di buon costume estemo sono o tuffi [li Gesuiti], o la maggior parte. La morale hanno rilasciata e contraria all'Evangelio, perché hanno volute esser potenti, $e$ ricchi, aver gran seguito, e acquistarse ereditá dei lor penitenti lasciando che si enpiano di peccate. Questultimo lo veda Vostra Eccellenza -escribe al duque de Losada, acaso el hombre más cercano a Carlos III- nell'opinione che sostengono dell'attrizione bastante colla confessione a cancellare li peccati ciò̀ il pentimento pel solo timore deI'inferno e senza latto di amor di Dio's.

(...) Li Gesuiti poi al capezale d'un che sia pieno di peccaii, fanno l'altro fatto suo coll'insinuar di redimersi colla linosina cioé con lasciare loro.

Y no se trataba de una mera elucubración teológica. "Molti ministri togati si son difesi da me, che biasimava alcune esazione ingiuste m'hanno risposto che'l confessore gesuita gli ha assicurati perché facendo il Re far loro qualche straordinario non li paga onde si possono compensare". Y más: "non si scandalizzi s'io li dico che il gran concorso di dame, e donne ai Gesuiti confessori un dotto e santo vescovo mi ha affermato d'aver appurato che vien dall'opinione de' Gesuiti che li toccamenti, e li baci non son peccati, e giá si trova questa opinione stampata nelle opere di due Gesuiti"19.

Y luego estaba su influencia sobre el papado:

[...] nel Palazzo pontificio non si sentono altri, che Gesuiti, onde sempre si vorrá unire la causa della corte di Rona con quella de' Gesuiti2".

Ora [...] Roma é diretta in tutto e per tutto dalle cabale dei Gesuiti, e dal carattere ardito, e feroce, e brutale di Torrigiani ${ }^{2}$.

Il quarto voto che costoro fano di difender nuti li capricci, tutte le rapacitá, e tutti gli abusi che il Papa faccia alle Chiavi e tuthi ancora gli altentati dei Papi che si sa aver fatto congiure contro le vite dei principi, e molte sedizioni é un'ostilitá che li Ge-

17 A Galliani, 7 de noviembre de 1761 , ibiden (1988), X, 275.

IR "Uno che si astiene dal peccare pel timore della pena -sigue Tanucci- non é anico di Dio ma di sé stesso, $e$ in paradiso non posson entrare se non gli antici di Dio. l...J Jatto di amor di Dio non sta in nostra mano il farlo, deve essere una grazia di Dio; il timor dell 'inferno e un confessore non manca mai ed ecco che si pecca continuantente e allegramente perché si confida in quello che non manca; chi crede che li pectah non si cancellano senza l'atto d'amore, e sa che tal atto é una grazia rara di Dio e non sa se l'avrá reme di peccare, e perció pecca nemo."

19 A Losada, 3 de noviembre de 1761 , apud TANUCC] (1988), X, 258-9.

20) Tanucci a Wall, 25 de agosto de 1761, ibidem (1988), X, 43.

21 A Losada, 15 de septiembre de 1761, ibidem (1988), X, 116.

La Personalidad Internacional de los Papas

Hispania Sacra 48 (1996) 
suiti fanto a tutti li sovrani, a tutte le nazioni, e a tutti li stati. Sono essi perció e devono essere in stato ostile e tutti devono esser loro nemici, essendo essi nemici di tutti22.

Del cuarto voto, en suma, procedía "tutto il sistema iniquo della Dataria, $e$ Segreteria de' Brevi, tutte le strane opinioni del dispotismo papale, tutta la depressione delle sovranitá, tutte le insidie che continuamente da Roma si tendono alle nazioni, e alli Stati, di questa depravazione della religione, $e$ della societá é composto [...] il papato cioè il pontificato dei nostri tempi fondato da Ildebrando [Gregorio VII] sulla cecitá dei secolari e sulla trascuraggine de' governi secolari. Che vuol dunque che faccia un consiglier di Stato che si regoli col confessor gesuita? Gli altri, monici son piú o meno ma tutti sono sbirri, e spie di Roma, da cui aspettano le dispense, gli avanzamenti, e li vescovadi, e hanno contro li governi secolari l'interesse stesso di Roma, $e$ professano la sedizione medesima, lindipendenza, l'orgoglio, la rapacità papale ${ }^{23}$.

La Chiesa cattolica per molti secoli non conobbe altri santi che i martiri, siccome la moderna non da tale onore che a frati, e non vuol santificare né il cardinale di Turnon, né il venerabile Palafox, che sono li veri martiri modemi ${ }^{24}$.

Así, la maldad de los jesuitas había venido a coincidir con la de la curia romana, el secretario de Estado Luigi Torrigiani —deudo y pariente del general de los jesuitas, Lorenzo Ricci- y hasta el papa reinante, que era Clemente XIII. En el palacio pontificio reinaba un pensar torbido e maligno ${ }^{25}$; se trataba de "un pontificato ignorante, e imperito, [...] un Arlecchino finto principe"26, un pontífice "incapace, o per natura, o per incantesimo de' Gesuiti e di Torriagini della ragione, e di qualunque affare, e servo in vece di esser padro$n e^{r 27}$.

1... il Papa non é mai Melibeo, é Daneta ciò̀ un pastore mercenario del non suo gregge, e perció egli non esercita il precipitato con quell'anore col qual sogliono amministrarlo li sovrani ereditari28.

Il Papa imbecille col suo ministro stolidanente feroce, e brutalmente furbo, hanno presto, come cani, scossa la battitura. Urtano ciecamente in tutto, e con tutti2y.

22 A Galiani, 31 de octubre de 1761, ibidem (1988), X, 252-3.

23 A Caraccioli, 31 de octubre de 176l, ibidem (1988), X, 253.

24 A Cattolica, 8 de septiembre de I76], ibidem (1988), X, 93. Sobre Tournon, ibidem, en nota.

25 Tanucci a Centomani, 15 de agosto de 1761, ibidem (1988), X, 9.

26 A Centomani, 7 de noviernbre de 1761, ibidem (1988), X, 276.

27 A Orsini, 21 de noviembre de 1761 , ibiden (1988), X, 310.

28 A Centomani, 14 de noviembre de 1761, ibidem (1988), X, 294.

La Personalidad Internacional de los Papas Hispania Sacra 48 (1996) 
Capitata la $S$. Sede in mano a due mercanti quali son nati e educati, il Papa, e Torrigiani, non pensa che a danaro, e a pascer l'inerzia e ad esser una stalla di porci epicureik?.

[...] costui è insoffribile e serve un papa insoffribbile, quello per la rusticità, e igno. ranza orgogliosa, questo per l'imbecillità avarizia, e passione per li suoi che non hamo alcun merito, e fanno all'tinteresse loro privato servir l'apostolato di S. Pietro $\$$.

So che al re di Spagna non piace cotesto ponfificato. So che li piaceva molto quello di Lambertini32.

\section{(Se refería a Benedicto XIV.)}

(...) il Re ne sa infinitamente piú del Papa, non dico di politica sola, ma ancora di canoni, e di dottrina cristiana. Ci vuole altro che lagrine di cocodrillo, o di puttana, per far bene il Papa, e il segretario di Stato del Papa. Ci vuol buon cuore, disinteresse, teologia, prudenza, semplicità, e nobilità di pensare, e sopratuto carità cristiana, uniltà, fede, la quela manca certamente, ove mancano le opere buone, e perciò non so che mi credere del presente Pontificato 3 .

Roma è ridotta alla sola spelonca di Caco, e la legge di Cristo a ius dei Ciclopi, quali veramente son costí riusciti li papi veneziani ${ }^{34}$.

Como nuncio en España, "Torrigiani dovrebbe avervi un villano, un criminalista, un banchiere inesorabile come lai'35.

Torrigiani è la cloaca di cotesto imbecellissimo pontificato. Tutti gli escrementi di quello cadono in Torrigiani. Egli si dovrebbe accorgere che cotesti mercanti voglion farne il grazioso e l'utile, e a lui lasciame il duro, e l'odioso. Dicono li medici che l'animale piá debole produce naggior copia d'escrementi. Cotesti debolacci non potevan trovar vaso piu adatto di Torrigiani a ricevere ed a tirarse ancora tutte le indignazioni, e le fecce del pontificato ${ }^{36}$.

1... I se dura cotesto crininalista di Torrigiani, sarà abolita la dottrina di S. Paolo, S. Agostino, di S. Tonmaso, e stabilita quella di Pelagio, di Molina, di Busembaun etc. ${ }^{37}$.

29 A Montallegre, 15 de septiembre de 1761, ibidem (1988), X, 126

3) A Caraccioli, 31 de octubre de 1761, ibidem (1988), X, 254.

3 A Pignatelli, I de diciembre de 1761 , ibidem (1988), X, 335.

32 A Centomani, 5 de diciembre de 1761, ibidem (1988), X, 346.

33 A Centomani, 2 de enero de 1762, ibiden (1988), X, 42I.

34 A Centomani, 22 de diciembre de 1761 , ibidem (1988), X, 381 .

3 A Centomani, 14 de noviembre de 1761, ibidem (1988), X, 293.

36 A Centomani, 5 de diciembre de 1761 , ibidem (1988), X, 346.

37 A Centomani, 19 de diciembre de 1761 , ibidem (1988), X, 378

La Personalidad Internacional de los Papas

Hispania Sacra 48 (1996) 
Torrigiani è come li sordi, che parlano a voce alta perché credono sordi parimente quelli col quali parlano. Non sa la storia ecclesiastica, sa solo la giurisprudenza della Marca, e crede che li ministri de' principi sieno egualmente persunsi di questa, e ignoranti di quella. A questa ferocia aggiunge le cabele dei Gestiti ${ }^{38}$.

Egli superbo, e provisto di beni di fortuna non ha bisogno di una virfú laboriosa, ed eroica per essere onesto, e parerlo; poca riflessione, e poco stinolo dell'onore, e della coscienza dovrebbe bastare per contenerlo dentro li confini della giustizia, e della pudicizia. Questa tanta parzialità per li Gesuiti, ai quali serve fino all'assassinio, [...] è una poca cura del suo nome. Ognuno lo crede senza scrupolo stipendiato, e corrotto con danaro dalla scelerata Compagnia, la quale non può avere altri fautori che o sciocchi, che si lascino burlare dalla apparenze, o scelerati, che si lascino corrompere o permutino qualche altra malvagità con essa"s.

\section{LA MALDAD ESENCIAL DE ROMA Y LA PROFUNDIDAD DEL REGALISMO}

La verdad es que el papa Clemente XIII había sido saludado en la corte española de manera optimista en 1758, cuando fue elevado al solio. Pero enseguida se puso de relieve que los que rodeaban al pontífice querían reformar, si no derogar, el concordato suscrito con el rey de España en 1753, que marcaba la cota más alta alcanzada por el regalismo. Lo que pesaba más es que se reservaba en él a la Santa Sede la provisión de 52 beneficios tan sólo, y esto con tales condiciones que incluso en esos casos el monarca español podía interferir eficazmente. Esta actitud ante el concordato influiría notablemente en que se estableciera una neta distinción entre Benedicto XIV - papa en 1753-y el veneciano Clemente XIII en el ánimo de los que rodeaban a Carlos III. Del primero se destacaba su firmeza y sus conocimientos canónicos, en tanto del segundo se ponía de relieve su debilidad y la limitación de sus saberes. "Mudóse de baraja —escribía Gándara a Wall en 1758 desde Roma, ante la formación del nuevo equipo de gobierno-. [...] es necesario considerar ya este pontificado como un mundo nuevo"40.

Non solamente sará Torrigiani nemico di Spinelli -comentaba Tanucci ante un asunto concreto--, ma ogni Cardinale lo sará dellaltro. ' $E$ cotesto il vero paese de' Ciclopi e dei Centauri. Bisognano i Lapiti a piantavi la ragione, e l'tancanitá, e la clava d'Ercole. Dopo, che cotesta gente si è unita a guastar colle sue furberie, abusando della Religione, il mondo cristiano, rimane che guasti la sua stessa Casa, e le rinscira, come successe a Roma antica. Una cospirazione di malvagità non può funire altrimenti che con divorar se stessa. Chi riflette in tua occhiata alla storia vede qutanto siano vicini a tal distruzione. La caduta è cominciata da Leon X Eresie, Concili, Concordati, Exequa-

38 A Wall, 15 de septiembre de 1761 , ibidem (1988), X, 118.

3 A Bottari, 21 de noviembre de 1761, ibidem (1988), X, 308-9.

49) Cit. MAClaS (1994), 717. Sobre las reservas ante el concortado de 1763, ibidem, 659-93. 
ur sono li vermi di questa putredine, la quale essa stella li ha generati. Da qualche tempo si è aggiunta la vanită, e l'ignoranza, né Antonelli può esser buono, né alcun altro che voglia e abbia voluto esser cardinali ${ }^{41}$.

Roma è stafa dopo li dieci secoli sempre donna —añade, después de asegurar que a las mujeres les gusta la afirmación de la moral jesuítica de acuerdo con la cual "oscuła, et tactus non son peccati"-, secondo l'Apocalisse, interpretata anche in lingua italiana da Dante, il quale con S. Giovanni, a cui si paragonò l'Ariosto, la chiana con un vocabolo anche peggiore di donna. Ella è potente costí per li cardinali, [... 1. Li sovrani non vogliono accorgersi della peste di questi sacri ribelli, $e$ sediziosi, li quali all'ombra della religione stanno in mezzo alli stati, $e$ alle corti in atto sempre ostile, e membri, $e$ strumenti di un corpo estraneo, che rode lo stato"42.

La misoginia de la concepción regalista aún brotaría con más fuerza al equiparar los eclesiasticos a las mujeres:

[...] listessi mali, le stesse anti -resume Tanucci--, e perché nulla manchi nel paragone la natura ha voluto che le femmine appartengano più alli secoli futuri che al presente, poiché appartengono alla propagazione della specie, e non devono poter calcolar colli mali del parto li piaceri della generazione, onde sono state proviste dalla natura di passioni inpetiose, e d'entusiasno invece di raziocinio, deboli in sommo, furbe, lusinghiere, trasportate, e senza legge, né fede vogliono non solanente vivere, ma lussireggiare senza fatica delle fatiche, $e$ de' beni altrui. Inquietano, sconfinano, offendono, usurpano, violano leggi, insidiano, e trovati rei esclamano violenze, $e$ oppressioni non solamente il gastigo, ma ancora l'innocente, e caritatevole avvertimen$t o^{4.3}$.

(...) Finalmente lo stesso fanno le fenmine delle Corti; destinate a partorire hanho la passione in vece del razziociniot4.

Frailes, monjes, regulares: "Questa generazione d'animali son troppo lontani dalla natura, e dall'umanita, sono li veri Genovesi di Danti, uomini diversi d'ogni costume, e pieni d'ogni magagna, e non si sa perché non sieno dal mondo spersi ${ }^{\prime 4}$.

No era, sin embargo, un problema circunstancial, pasajero, vinculado a la desgracia de tener un papa como Clemente XIII. Era la historia misma de la Iglesia, la Iglesia "histórica" la que encerraba el mal.

41 A Centomani, 22 de agosto de 1761, apud TANUCCI (1988), X, 36.

42 A Galiani, 29 de agosto de 1761 , ibidem (1988), X, 61.

43 A Caraccioli, 19 de septiembre de 1761, ibidem (1988), X, 132.

44 A Galiani, 5 de diciembre de 1761 , ibidem (1988), X, 344

4 A Centomani, 14 de noviembre de 1761, ibidem (1988), X, 293.

La Personalidad Internacional de los Papas

Hispania Sacra 48 (\$996) 
Se Roma non ammette tralli delitti de' Gesuiti le cospirazioni, è perché la professione di Rona è il delitto continuo di lesa maestà conninciato forse da S. Cirillo, e forse prima $a^{46}$

L'unione della sovranitá temporale che Nostro Signore Gesù Cristo aveva proibito all'Apostoli ed a sé stesso, coll'apostolato ha prodotta nei papi una condotta politica colla confusione artificiosa di corte, e di religione, e per lo piu si è fafta servir la religione alla grandezza sovrana. Hanno li papi fatte le guerre, hanno suscitato sedizioni nelli Stati de' principi secolari, hanno fatto congiure contro la loro vite, come la storia mostra in Leon IV, Gregorio VIII, Innocenzo III, Bonifazio VIII, Sisto IV, e forse Sisto $V$. Paolo $I V$ etc. etc. Facendo poi le paci han fatto uso dei lor vantaggi di sovranità, hanno fatfo concordati secondo il vento a favor del papato, introducendo linvenzione delle pensioni ecclesiastiche su' vescovati, prendendosi li patronati regi, mettendo in commenda li monasteri, usurpandosi le nomine de' vescovati $[. .$.$] , introducendo le tas-$ se, le annate, li spogli, e mille alire cose contrarie non meno alla giustizia ai poveri de quali sono i beni di Chiesa, alli Stati, ai sovrani che all'Evangelio, alla tradizione apostolica, alla disciplina della Chiesa ${ }^{4}$.

Noi italiani, che sappiamo cone penșano li nostri preti, dei quali $\dot{e}$ la maggior parte della corte di Roma, non ci maravigliano, di che tal Corte trati la religione, come un bene temporale, che si deve stendere colla guerra ancora. Son dieci secoli, che la corte di Roma tiene questa opinione, e opera con essa; le crociate, e gli ordini militari, e il S. Offizio, e tante invenzioni sono state o archifettate, o favorite dalla corte di Rona; percio non è da maravigliarsi, che si opponga all'anicizici, che un sovrano cristictno tratti con un altro infedele. Son inaudite in Roma, le massime sante, di che la nostra santa religione fondata dal divin Redentore nella fede, nella caritá, nella speranza, neI'umiltà non deve esser sanguinaria, e deve propagarsi con quelle virtì, non colla spada, e si devon convertire gl'infedeli coll'esempio e colla preghiera a Dio, non col cannone, che finora non ha convertito nessuno ${ }^{48}$.

\section{De ahí la necesidad del regalismo:}

1... Ii sovrani cattolici a forza d'una lunga esperienza e tolleranza si sono accorti del pretesto di religione, che la corte di Roma è asuefatta ad usare per coprive la sua infinita rapacità di giurisdizione, e di danaro. Scrivono, e parlano, colli sovrani sullo stile solito anche dopo che si è imparata l'arte di separare la religione cattolica dalle astuzie, e interessi profani di quella Corte. La storia pura della Chiesa è stata l'acqua regia di quella divisione, che la stessa conte di Roma ha resa necessaria coll'abuso eccessivo portato all'estremo della sofferenza secolare4).

'E inutile parlar di Genova, e di Corsica come di Portogallo per pacificazione. Le nazioni vanno assuefacendosi ad esser cattoliche senza Roma. [...] Essendo illuninate le carte per colpa di Roma la quale ha messo la gente colle spalle al muro, è facile ri. durre il papato ad esser quel che deve, dopo che è stato dichiarato l'abuso di voler

\footnotetext{
46 A Galiani, 27 de febrero de 1762, ibidem (1988), X, 572 .

47 A Losada, 3 de noviembre de 1761 , ibidem (1988), X, 259.

48 A Wall, 5 de enero de 1762 , ibidem (1988), X, 429 .

49 A Wall, 15 de septiembre de 1761 , ibidem (1988), X, 118.
}

La Personaliơd Internacional de los Papas Hispania Sacra 48 (1996) 
esser quel che non doveva ciò il despotico, capriccioso delle cose umane e divine in vece di esser un capo maestro del domma e del rito, a cui dovesse prestarsi dalli fedeli I'ossequio ragionevole al quale solo son obbligatis".

Y ante la reacción defensiva de los príncipes, de nuevo el recurso a la moral laxa de los jesuitas, que así acababa de cumplir su nefasto papel: "Questo [el regalismo] dispiace ai papi, e per mezzo dei Gesuiti nelle Corti, nelle confessioni, nelle consulte, nei colloqui insinuano da per tutto massime contrarie alli sovrani, e alli Stati, e ove possono colla voce e co' libri van dicendo che Gesú Cristo dà al Papa la potestà di sciogliere, e legare la quale essendo da Gesí Cristo data a tutti gli Apostoli poco prima dell Ascensioni pel foro penitenziale, li Gesuiti ristringono al solo papa, e per altra parte l'allargano al foro esterno ancora, e fanno il papa gitudice de' sovrani li quali possa punire, togliere, e mettere come a lui piace. Queste dottrine predicano, queste insegnano nelle scuole [...]. Lascio la dottrina sacrilega della grazia contro S. Paolo. S. Tommaso, dalla quale nascono tante eresie. Lascio la politica di far tutti gli sforzi perché la Scrittura Sacra, e particolarmente il Testamento Nuovo non si traduca nella lingua volgare perché la gente volgare non veda quanto all'Evangelo, e all'Epistole degli Apostoli è contraria la lor dottrina. [...] Lascio il voler con tante confraternite di dame, di cavalieri, di servitori, di artisti, di dottori saper tutto, $e$ tutto riferire al Generale, e al papa, il pubblico, e il privato delle nazioni, li segreti delle Corti, gl'interessi delli Stati"si.

Del fondo de su anticurialismo, surge en realidad un jansenismo próximo al ideario calvinista en algunos aspectos. "Ella -dice en otra ocasión a Domenico Caracciolo, representante de Fernando de Nápoles en Turín- mi avverte a guardarmi [...] da quello stile della Curia romana, in che le anime semplici, e portate dietro alla verità, alla giustizia al bene de' popoli, e de' sovrani, devono necessariamente incorrer per l'odio de' furbi, de' falsi, importuni ipocriti, che avari, e ambiziosi, e senza merito vero verso il genere umano per arricchire, ed essere onorati si attaccano alla divinità, e fingendosi interpreti, e dispositori ingannano la gente, e si fanno pagar caro quel paradiso, che promettono a chi da loro lo compra col danaro, col seguito, collobbedienza, e cieca subordinazione. Ma io son persuaso della grazia effica$c e$, della predestinazione, del fato, e di non essere noi altro che machine condotte pel temperamento, pel piacere, per uno trahit sua quemque voluptas, ove l'autor supremo della natura ha disposto. Corro, e correrò il corso mio. Rin.

51 A Centomani, 21 de noviembre de 1761, ibidem (1988), X, 310.

s) A Losada, 3 de noviembre de 176), ibidem (1988), X, 259-60.

La Personalidad Internacional de los Papas Hispania Sacra 48 (1996) 
grazio questo autore della natura, che non mi ha messo sul cammino dell'avarizia, dell'ambizione, della menzogna"52.

Pero el regalismo era preciso en sí, para acabar con la usurpación del poder de los príncipes que se había llevado a cabo desde la jerarquía eclesiástica.

'E drifto delle genti questa regalia - decía concretamente sobre el exequaturSomma grazia è che li vescovi possan fare editti. II farle è nuova usurpazione che vescovi han fatto nello Stato. La lor giurisdizione furor di quella delle censure è tutto dono, indulgenza, e condescendenza de' principi secolari. Questa grazia invece di riscuoter gratitudine è abusata e convertita in insolenze, e querele, e calunnie ancora [... J'E favoloso l'ordine ecclestasticos?.

Giurisdizione, interesse, e abuso della religione è tutto l'affare degl'ecclesiastici nel mondo cattolico 5 .

Li vescovi hanno da Dio la facoltà di predicare e di conoscere colla Chiesa cioè col sinodo se una censura abbia luogo; finalmente l'imposizione delle mani, $c$ il foro penintenziale. Giurisdizione e potestá è quanta ne hanno permissione o espressa delegazione de' principi cristiani, li quali possono a loro arbitrio riformarne l'abusi e ripigliarse$10^{55}$.

Doctrinalmente, la cosa estaba clara a su juicio:

Questa [Rona] dice che Ii vescovi hanno da Gesí Cristo le chicrvi del Regno de' Cieli, cioè il foro penitenziale, ed hamo dai sovrani catolici la giurisdizione del foro esterno sulli preti ed altri ecclesiasticis.

Il domma e il foro penitenziale è tutto della Chiesa cattolica apostolica, [...] la giurisdizione esterna, e il foro estemo degli ecclesiastici è tutta grazia, e concessione loro fatta dai sovrani cattolici. Nei benefizi ecclesiastici la Chiesa ha privativanente l'imposizione delle mani cioè l'ordine sacro, $l i$ beni son parinente concessioni laicali sottoposte alla sovranità, che non puo alienar né la sua giuristizione, né la protezione che deve ai sudditi, allo Stato, $e$ a se stessa ${ }^{57}$.

No era esto lo que había. "[..] col pretesto di religione essi []os eclesiásti$\cos$ ] insidiano, $e$ invadono e beni, e giturisdizione e regalia come l'esperienza lunga dimostra"58.

$\$ 2$ Carta de 5 de septiembre de 1761, ibidem (1988), X, 83 .

53 A Orsini, 26 de septiembre de 1761 , ibidem (1988), X, 158.

34 A Squillace, 29 de septiembre de 1761, ibidem (1988), X, 165.

ss A Orsini, 3 de octubre de 1761 , ibidem (1988), X, 174.

sh A Losada, 27 de octubre de 1761, ibidem (1988), X, 242

57 A Wall, 27 de octubre de 176I, ibidem (1988), X, 244.

58 A Wall, 29 de septiembre de 1761, ibidem (1988), X, 172.

La Personalidad Internacional de los Papas Hispania Sacra 48 (1996) 
Sono infinite le usurpazioni degli ecclesiastici contro le nazioni, e la sovranità tese, e fatte softo il pretesto di religione $e$ anche di dogma. Danaro e giurisdizione, non religione è l'oggetto principale degli ecclesiastici. Della religione si vagliono come di strumento, e di mezzo e se per ottener danaro e giurisdizione stimano che lor convenga mutare il dogma anche quello muttano, [...]. Il buon cattolico è quello che crede, conte dice San Giovanni Crisostomo, quello che si è sempre creduto da futfi, e in tutto il mondo cattolico. Ma li delusi e ignoranti celano le loro tenebre e le scusano con una bruta* le credulità, e se ne vantano benché San Paolo abbia deto che l'ossequio alli superiori ecclesiasici deve esser con ragione sit autem rationabile obsequium vestrum. Se fale deve essere, viene in conseguenza [...] che si deve esaminare quello che it prelati ci dico. no, e piú si deve quanto piú la lunga esperienza ha insegnato che li ecclesiastici cibusano per sordide, e profane loro passionit5.

(...) non posso negare alla mia coscienza l'esperienza di non aver mai veduto gli ecclesistici peccare in favore delle sovranità, come sarebbe qualche volta accaduto, se generainente procedessero per ignoranza. Sempre peccano col pretender piti di quello che lor si deve, non mai col pretendere meno. Peccano sempre di poco rispetto ai so. vrani, e ai magistrati; sempre cabale, e fraudi alle giurisdizioni e aziende del principe; sentpre insidie alla regalia, al patronato, alla preeminemza sovrana. Sempre avari, sempre fastosi, sempre contrari alle leggi, e alla disciplina dello Stato; sempre confinanti colla sedizione. Non riescono ignoranti in questa loro intrapresa che è ridotta a professione diabolica, ma riescono sottili, e sofistici, come sottili e accorti riescono nell'inventar tasse, spogit, annate, dispense, e altri artifizi infenali per far danaro, $e$ in danaro convertire quotidianantente li sacri canoni, e le massime fondamentali della disciplina cristiana, e de' concili. Sarà dunque ignoranza quella colla quale ci danno continue molestie, ma è una molto per loro profittevole ed hamo ragione di conservarsela, e molto piú ragione hanno di stabilirla per quanto possono nei secolari. Questi perché erano veramente ignoranti ne' i secoli tenebrosi hanno lasciato agli ecclesiastici far tante usurpazioni di beni, e di giurisdizione profana, e stabilire un regno temporale nei loro stati, e per sete de' bent temporali abusare della spirifuale autoritìt.

Pasara que el pontífice se atribuyera títulos como el de la infalibilidad, siempre que quedaran precisamente en títulos, pero no si, además, servían para sembrar la injusticia en todos los actos.

Linfallibilità, che il Papa suol sostenere, conte un titolo di re d'inghilterra, $e$ Francia, di sposa del mare Venezia, i re di Gerusalemme el re delle Sicilie, d'Imperatore dei Romani el re di Germania, si potrebbe lasciar correre, se non si esigessero $1 i$ dritti di quella infallibilità, cone il Papa pretende di fare. Titolo vano passi, ma titolo ingiusto, che mi si porti per spogliami del danaro, della giurisdizione, della libertà, della ragione, se io non resista, sono uno sciocco, e un pusillanine ${ }^{\text {al }}$.

59 A Cattolica, 29 de septiembre de 1761, ibidem (1988), X, 163.

*1) A Wall, 13 de octubre de 1761, ibidem (1988), X, 205-6.

ol Tanucci a Galiani, 22 de agosto de 1761, ibiden (1988), X, 35.

La Personalidad Internacional de los Papas

Hispania Sacra 48 (1996) 
S. Paolo dice che alla Chiesa si deve obsequium rationabile cioè non cieco, né brutale. Il Papa non è sovrano, è maestro della religione, e il maestro deve illuminarét.

La Chiesa è nello Stato, e sullo Siato, se non se le apre la porta non può entrare; la porta si apre col yoler del Principe, o con un esercito vittorioso ${ }^{63}$.

\section{LA COYUNTURA REGALISTA DE LOS AÑOS SESENTA}

A los ojos de aquellos regalistas, había venido a darles la razón, entre otras cosas, la reedición del catecismo del concilio de Trento -el de San Pío Vque había ordenado hacer Clemente XIII en 1761 y del cual el jesuita Giambattista Faure - activo polemista, también beligerante contra la beatificación de Palafox- expurgó o sustituyó los pasajes y citas de los Padres de la Iglesia que menos convenían a la orientación actual del pontífice, concretamente las que implicaban el reconocimiento de la autonomía de los obispos en el ejercicio de la jurisdicción, en relación con el papado, así como las que podían interpretarse contrarias a la doctrina jesuítica sobre la gracia y la laxitud moral. "La corruttela del catechismo romano -comentaría Tanucci- è sporca, scandalosa, e scismatica, poiché la Chiesa universale non è nelle Bolle romane, ma ne' concili, dei quali fu il catechismo romano la voce fedele approvata, e celebrata, e predicata da Roma stessa prima che tante furberie la guastassero"64.

$\mathrm{Si}$ a ello aún sumamos la especie de que los jesuitas tenían especial malevolencia del rey Carlos III, termina de entenderse la expulsión: según afirmaciones de Tanucci, habían procurado cuanto fuera posible evitar la entronización del monarca napolitano, o al menos dilatarla. Y, cuando llegó a España y comenzó por conseguir que se reabriera el proceso de beatificación de Palafox, en 1760 , jesuitas habían sido quienes corrieron la especie de que el nuevo monarca y sus ministros eran herejes y que la religión estaba en peligro, aparte de dar pábulo a profecías misteriosas según las cuales el reinado no duraría más de seis años ${ }^{65}$.

Enseguida había venido la condena romana del catecismo de Mésenguy, que dio lugar a la reimposición del exequatur y que Tanucci presentó al rey de España como maniobra jesuítica: "[...] gesuitica è stata probabilmente questa

62 A Losada, 8 de septiembre de [761, ibidem (1988), X, 97.

6. A Centomani, 12 de septiembre de 1761, ibidem (1988), X, 108.

64 A Orsini, 29 de agosto de $176 \mathrm{~J}$, ibidem (1988), X, 60. Sobre lo mismo, ibidem, 7, 18-9, 92 , 116-7 y passim. Subraya la importancia del antitregalismo, como causa de la expuisión, BATLLORI (1985), 366-8.

65. Todo esto según Ferrer (1856), I, 260-1. Que Carlos III tenín idea clara de la opinión de Tanucci sobre los jesujtas, se desprende con claridad de ibidem, 184-6. 
[cabala] armata, e promossa da Torrigiani. [...] Votra Eccellenza -escribe a Wall- si serve di rammentarmi a questo proposito, la mia tragica profezia di che il trionfo de' Gesuiti sulla proibizione del catechismo, causerebbe disturbi in Europa. Non avrei però creduto, che tal molestia venisse a Spagna"6r. 'Tutto è matassa, e cabala de' Gesuiti, de' quali sono in cotesti magistrati e chiese tante creature" quel che hanno fatto contro il povero Mesangui? Non son dunque arrivati ancora alla virilità, che commisisse cavet quod mox mutare laboret, $e$ sono ancora nello stato dello sfrenato giovinastro sublimis, cupidusque, et amata relinquere pernix, o per dir meglio in quella puerilità vergognosa, che iram colligit, et ponit temere, et mutatur in horas, che non si perdona, se non ai visi lisci, ed inesperti"68.

Lasciate fare - comentaba al abogado y amigo Gaetano Centomani ante una intervención semejante, concretamente contra un libro de moral del dominico Castellana-; sempre piú argomenti contro l'infalibilità del Papa, sempre pia pritove del dispotismo, e del capriccio del papato, onde son li concili necessari, sempre pili martirio de buoni, che è la via regia per la propagazione $e^{69}$.

Y en 1762-1763, la separación de los jesuitas que tenían el cargo de confesar al príncipe de Asturias y a los infantes, y esto porque tuvieron la osadía de quitarles a éstos las obras de Palafox, recién impresas, que acababa de regalarles el monarca su padre ${ }^{70}$. Antes de partir para España en 1759, ya había Carlos III encargado a Tanucci que no dejase solo a jesuita alguno con el rey niño de Nápoles, y esto contra el filojesuitismo de la reina Amalia de Sajonia ${ }^{71}$.

"Puesto que perdían los jesuitas o los colegiales ya no volvían a recuperarlo -diría Ferrer del Río ${ }^{72}$-: cuantos claros dejaba la muerte en las diversas carreras, llenábalos el monarca a tenor de sus miras". Cosa que se acentuó en 1765 con la muerte del marqués de Campo del Villar, a quien sabemos colegial y valedor de los jesuitas ${ }^{73}$.

El rey estaba convencido de la maldad de los buenos padres, y el pueblo no iba a ofrecer resistencia porque muchos opinaban lo mismo. Tras la caida de

7

66 A Wall, 1 de septiembre de 1761, apud TANucCI (1988), X, 66. Vid. FerRer (1856), 1, 386-

67 A Losada, 8 de septiembre de 1761 , apud TANuCCI (1988), X, 97.

6* A Bottari, 26 de septiembre de 1761, ibidem (1988), X, 152-3.

69 Carta de 2 de enero de 1762, ibidem (1988), X, 421.

70 Cfr. FERRER (1856), I,397.

$"$ Vid, sobre esto TANUCCI (1988), X,137, 178.

7 En Ferrer (1856), 1,413.

73 En este sentido, FERRER (1856), [,425.

La Personalidad Internacional de los Papas

Hispania Sacra 48 (1996) 
Rávago como confesor de Fernando VI, en 1755, había habido una primera invasión de libelos antijesuíticos, donde se les culpaba de todo lo culpable. De hecho, en 1759, varios prelados se consideraron obligados a salir en su defensa públicamente y el propio papa lo hizo con la constitución apostólica Apostolicum pascendi de $1765^{74}$.

La expulsión de Portugal y de Francia, además, había sido un precedente efectivo para crear el clima de irreversibilidad de la expulsión. Tres años antes de que se consumara en Francia ya se daba como segura. "Le lettere di Francia son tragiche per li Gesuiti -escribe Tanucci en agosto de 1761- Cantillana scrive, che el golpe es ireparable [sic] [...]. I libri de' teologi de' Gestiti in una prodigiosa quantità son già stati bruciati solennemente per man del boja, e li decreti sono stati intimati, e pubblicati gli editti'75. Se refería a dos decretos del parlamento de París del 6 de agosto: en uno de ellos se condenaban veinticinco libros jesúticos publicados desde 1590 y en el otro se prohibía entrar en la Compañía de Jesús a cualquier súbdito del rey de Francia y que los jesuitas enseñaran teología, filosofía o humanidades pública o privadamente. Tenían, pues, que cerrar sus colegios, seminarios y escuelas.

II Parlamento di Parigi ha fatto contro li Gesuiti un principio di vendetta del genere unano -añade aún Tanucci-. Il principio promette molto seguito, perché è sonoro, e prende molta estensionte. [...] hit Firenze voglion fare una festa pubblica sulla tomba del Galileo ${ }^{76}$.

Pagheranno li Gesuiti le pene del trionfo, che colle loro cabale hamo ottento in Roma colla proibizione del catechismo di Besanqui [sic] tradotto e stampato in Napoli coll'approvazione del Re Cattolico nostro Signore I'ano 1758 e con quella del Cardinal arcivescovo 7 .

Pero ¿podemos asegurar que pensaba así Carlos III, además de que lo hicieran varios de sus ministros? Por lo menos cabe decir que en junio de 1767, y por excepción, autorizó a Roda a explicar a Tanucci las razones de la expulsión y que lo que escribió el aragonés al italiano fue lo siguiente:

De todo [lo averiguado en la pesquisa secreta] resultó que los jesuitas eran el principal o único fomento que atizaba el fuego por todas partes, indisponía los ánimos de toda clase de gente, los separaba del amor y subordinación al Gobierno, imprimían y esparcían papeles y escritos sediciosos y contrarios a la autoridad real y a la soberanía y sus

74 Sobre los libelos y la defensa de algunos obispos, ALCARAZ (1995),707.

75 A Orsini, 29 de agosto de 1761 , apud TANuCCI (1988), X, 58. Lo que sigue, ibiden. El condc de Cantillana, Giusseppe Baeza i Vicentelo, era el embajador del rey de Nápoles en la corte francesa.

76 A Centomani, 29 de agosto de 1761, ibidem (1988), X, 60

I) A Cantillana, 29 de agosto de 1761, ibidem (1988), X, 61.

La Personalidad Internaciomal de los Papas Hispania Sacra 48 (1996) 
legítimos derechos, predicaban contra Portugal y Francia en sus sermones al público, y en los locutorios de las monjas, introduciéndose a dirigirlas y confesarlas sin autoridad, y contra la voluntad de los superiores de los conventos; infundían hasta en los claustros de las religiosas en sus pláticas y conversaciones máximas perjudiciales, y sospechas indignas contra la Religión del Rey y sus Ministros; amenazaban desgracias y tragedias en tono de profecías antes y después del tumulto. Se quejaban de todas las provisiones que se hacían por no recaer los empleos y dignidades en sus parciales y secuaces de su escuela. Mormuraban [sic] de todas las providencias del Gobierno, y porque no tenían parte en ellas, y por ser contrarias a sus ideas y ventajas. Su mala moral práctica en España e Indias, la relajación de sus costumbres, su sórdido comercio, sus intrigas, sus manejos, y por fin todo cuanto se ha escrito y publicado por los que la Compañf́a supone érmulos y enemigos suyos, se ha verificado y convencido con hechos y casos particulares modernos e innegables, sin necesidad de acudir a los muchos y enormes excesos de los tiempos pasados, ni de los países estranjeros [ $\mathrm{sic}$ ]. Su odio a la casa de Borbón, y su aversión al pacto de Familia, su parcialidad por los ingleses, y sus deseos de que ćstos oprimiesen a la Francia, la mayor satisfacción y confianza que ponen en los Príncipes protestantes prefíriéndolos a los católicos, y otros objetos abominables y contrarios al espíritu de religión, de honor y de humanidad, se han comprobado por muchos medios irrefragables, que nos han subministrado sus propios hechos, dichos y escritos ${ }^{78}$.

78. Carta de 23 de junio de 1767, facsímil apud DÁNVILA (1893), 111, 77-8.

La Personałidad Internacional de los Papas

Hispania Sacra 48 (1996) 


\section{BIBLIOGRAFÍA MENCIONADA}

ACEVEDO (1969), EDBERTO OSCAR: La rebelión de 1767 en el Tucumán, Mendoza, Universidad Nacional de Cuyo, 223 págs.

AlCARAZ (1995) GómEZ, JosÉ: Jesuitas y reformistas: el padre Francisco de Rávago (1747-1755), Valencia, Facultad de Teología San Vicente Ferrer, 795 págs.

ARManj (1982), Alberto: Ciludad de Dios y ciudad del sol: el "Estado" jesuita de los guaranfes (1608-1768), Méjico, Fondo de Cultura Económica, 232 págs.

Barajas (1994) Salas, Eduardo: "Cartas de académicos de la Historia al obispo de Beja, Frey Manuel Do Cenáculo": Boletín de la Real Academia de la Historia, CXCl, núm. 3, 517-564.

BATLLORI (1985), M.: "I problemi culturali e poititici dei Gesuiti sotto Carlo di Borbone in Napoli e in Spagna: vecchie e rinnovate prospettive storiografiche", cn I Borbone di Napoli e i Borboni di Spagna: Un bilancio storiografico, a cura di Mario Di PINTO, Nápoles, Guida, pág. 365-374.

BurRus (1963), ERNEST J.: Misiones norteñas mexicanas de la Compañía de Jesús, 1751 -1757, Méjico, Porrúa, 132 págs.

DÁNVILA (1893) y COLlado, MANuEl: Historia general de España escrita por individuos de número de la Real Academia de la Historia bajo la dirección del Excmo. Sr. D. Antonio Cánovas del Castillo, t. XII, Reinado de Carlos $H /$, vol. 3, 692 págs.

ESCANDÓN (1983), J. de: "Historia da transmigraçâo dos Sete Povos Orientais", Pesquisas, XXIII, 423.

FerRer (1990) BenjmeL.1, J. A.: "La expulsión de los jesuitas del Paraguay (según fuentes diplomáticas francesas)": in Estudios sobre la expulsión y extinción de los jesuitas, San Cristóbal, Universidad católica del Táchira, pág. 39-55.

FERrer (1856) DEL RIO, ANTONIO: Historia del reinado de Carlos II/, Madrid, Imprenta de los señores Matute y Compagni, 4 volúmenes.

KRATZ (1954), G: El tratado hispano-portugués de limites de 1750 y sus consecuencias, Roma, Istituto Storico della Compagnia de Gesu, 312 págs.

MaCías (1994) DELGado, JaCinTA: La Agencia de Preces en las religiones Iglesia-Estado español (1750-1758), Madrid, Ministerio de Asuntos Exteriores, 783 págs.

MATEOS (1951-2), F.: "El Tratado de Límites...", Missionalia Hispanica, VIII (1951), 241-316, y (1952), 75-121.

MEDINA (1991), FRANCISCO DE BORJA: "Ocaso de una provincia de fundación ignaciana: la Provincia de Andalucía en el exilio (1767-1773)": Archivo teológico granadino, LIV, 5-90.

La Personalidad Internacional de los Papas Hispania Sacra 48 (1996) 
NAVARRO (1908), EDUARDO: Documentos indispensables para la verdadera historia de Filipinas, con prólogo y anotaciones de..., Madrid, Imp. Asilo de Huérfanos, 2 tomos.

POlgÁr (1985), LÁszló: "Bibliographie sur l'histoire de la Compagnie de Jésus", Archivum historicum Societatis lesu, LIV, núm. 108, 377-468.

-(1986): Bibliographie sur l'histoire de la Compagnie de Jésus, 1901-1980, t. II, Les pays: Amerique, Asie, Afrique, Océanie, Roma, Istituto Storico della Compagnia de Gesù, 537 págs.

SANTOS (1992), ANGEL: Los jesuitas en América, Madrid, Mapfre.

TANUCCl (1980-1985b), BERNARDO: Epistolario, diretto da Mario D'ADDIo, tomos I-V y LX, Roma, Edizione di Storia e Letteratura:

t.I, a cura di R.P. CoPPINI e R. NeRI, Prefazione de Mario D'ADDIO (1980), LXXX +960

t. II, a cura degli stessi (1980b), 801 págs. t. III, a cura di A.V. MigliorinI (1982), xxv + 540 págs.

t. IV, a cura di L. DEL BIANCO (1984) LXXIX + 578 págs.

t. V, a cura di G. DE LUCIA (1985) LXXI + 951 págs.

t. IX, a cura di M.G. MAIORINI (1985b) Ll + 977 págs.

— (1988-1990): t. X y XI, Roma, Istituto Poligrafico e Zecca dello Stato:

t. X, a cura di M.G. MAIORINJ (1988), XXXIII + 686 págs.

t. XI, a cura di S. LoLlini (1990), XL + 849 págs.

- (1994): t. XIII, a cura e con introduzione di M. BARRIO, Nápoles, Società Napoletana di Storia Patria, 455 págs.

La Personalidad Internacional de los Papas

Hispania Sacra 48 (1996) 